\title{
Simultaneous LC-MS/MS Determination of Sacubitril, Valsartan and LBQ657 in Human Plasma and Its Application to Clinical Study
}

\author{
Yufeng Ni, ${ }^{\#, a}$ Yujia Zhang, ${ }^{\#, b}$ Chong Zou ${ }^{*, c}$ and Li Ding ${ }^{\odot *, b}$ \\ ${ }^{a}$ Yangtze River Pharmaceutical (Group) Co., Ltd, 225321 Taizhou, China \\ ${ }^{b}$ Department of Pharmaceutical Analysis, China Pharmaceutical University, 211198 Nanjing, China \\ 'Jiangsu Province Hospital of Chinese Medicine, Affiliated Hospital of Nanjing, \\ University of Chinese Medicine, 210029 Nanjing, China
}

\begin{abstract}
A rapid and reproducible liquid chromatography-tandem mass spectrometry (LC-MS/MS) method was developed and validated to simultaneously determine sacubitril, valsartan and a metabolite of sacubitril (LBQ657) in human plasma using sacubitril- $d_{4}$ and valsartan- $d_{3}$ as the internal standards. Following protein precipitation, the analytes were operated on an Ultimate ${ }^{\circledR}$ XB-C18 column $(2.1 \times 50 \mathrm{~mm}, 3.5 \mu \mathrm{m}$, Welch $)$ with a gradient elution with acetonitrile, and $5 \mathrm{mM}$ ammonium acetate and $0.1 \%$ formic acid in water as the mobile phase. The detection was performed on a Triple Quad 4000 mass spectrometer coupled with an electrospray ionization source (ESI) under positive-ion multiple reaction monitoring mode. The linearities are 2.00-4000, 5.00-10000 and 5.00-10000 $\mathrm{ng} \mathrm{mL}^{-1}$ for sacubitril, valsartan and LBQ657, respectively. The accuracy and precision of intra- and inter-day, dilution accuracy, recovery and stability of the method were all within the acceptable limits and no matrix effect or carryover was observed. The suitability of the method was successfully demonstrated in terms of the quantification of sacubitril, valsartan and LBQ657 in plasma samples collected from healthy Chinese volunteers in a clinical trial.
\end{abstract}

Keywords: sacubitril, valsartan, LBQ657, pharmacokinetics, LC-MS/MS

\section{Introduction}

As a new epidemic of cardiovascular disease, heart failure (HF) affects more than 37 million people worldwide. ${ }^{1,2}$ Associated with high hospitalization rates and mortality, HF has caused very significant health and financial burdens on patients, their families, and society. ${ }^{3,4}$ Sacubitril-valsartan, a first-in-class angiotensin receptor blocker-neprilysin inhibitors, represents significant reduction in the rate of hospitalization and mortality, and increases quality of life and ejection fraction. ${ }^{5,6}$ It is a novel single molecule comprising molecular moieties of the angiotensin receptor blocker valsartan and the neprilysin inhibitors prodrug sacubitril, which is rapidly hydrolyzed by esterase to an active metabolite of sacubitril, LBQ657.7-9

Several previous analytical methods have been reported for the determination of sacubitril and valsartan in rat plasma using liquid chromatography (LC) with fluorescence detector ${ }^{10}$ and liquid chromatography-tandem

*e-mail: zch816050@126.com; dingli@cpu.edu.cn

"Yufeng Ni and Yujia Zhang contributed equally to this work. mass spectrometry (LC-MS/MS). ${ }^{11}$ However, those methods only quantitatively analyzed the concentrations of sacubitril and valsartan. It is also essential to investigate the pharmacokinetics of the active neprilysin inhibitor LBQ657. Lately, a few articles in the pharmacokinetics and pharmacodynamics studies mentioned several LC-MS/MS methods for the determination of sacubitril, valsartan and LBQ657 in human plasma. ${ }^{12-14}$ However, those methods were only briefly mentioned without method validation results. Because of the evaluation of analyte stability, matrix effect in hemolyzed and lipemic plasma samples are required in recent regulatory guidance on bioanalysis, ${ }^{15}$ it is necessary to perform a more comprehensive method validation. Furthermore, the sample treatment methods reported were all liquid-liquid extractions which took relatively long sample preparation time. In this study, a simple and cost-effective protein precipitation method was applied instead of those liquid-liquid extractions.

This work presents a LC-MS/MS method for the simultaneous determination of sacubitril, valsartan and LBQ657 in human plasma, which was fully validated in accordance with bioanalytical method validation from 
FDA (Food and Drug Administration, USA, 2018). ${ }^{15}$ It was successfully applied to quantitate three analytes in human plasma samples collected from healthy Chinese volunteers after oral administration of commercial sacubitril-valsartan tablet in a clinical trial.

\section{Experimental}

\section{Chemical and reagents}

Sacubitril (purity, 96.9\%) was purchased from TLC (Mississauga, Canada). Valsartan (purity, 98.6\%) was obtained from the National Institutes for Food and Drug Control (Beijing, China). LBQ657 (95.6\%) was obtained from Suntech (Shanghai, China). Sacubitril- $d_{4}$ (purity, 98.6\%, internal standard for sacubitril and LBQ657) and valsartan- $d_{3}$ (purity, $98.6 \%$, internal standard for valsartan) were purchased from TRC (Toronto, Germany). HPLC-grade methanol, acetonitrile and dimethylsulfoxide were purchased from Merck KGaA (Darmstadt, Germany). Certified ACS grade ammonium acetate $\left(\mathrm{NH}_{4} \mathrm{Ac}\right)$ and formic acid (FA) were purchased from Sigma-Aldrich (St. Louis, MO, USA). Ultra-pure water was purified by a Milli-QTM system (Millipore, Bedford, MA, USA).

\section{LC-MS/MS instruments and conditions}

A Shimadzu liquid chromatography system coupled with a Triple Quad ${ }^{\mathrm{TM}} 4000$ mass spectrometer (Applied Biosystems/Sciex, USA) equipped with an electrospray ionization (ESI) source was used throughout the study. Chromatographic separation was achieved on Ultimate ${ }^{\circledR}$ XB-C18 column $(2.1 \times 50 \mathrm{~mm}, 3.5 \mu \mathrm{m}$, Welch $)$ equipped with a SecurityGuard ${ }^{\mathrm{TM}}$ cartridge $\mathrm{C} 18$ column $(4 \times 2.0 \mathrm{~mm}$, Phenomenex). The column temperature was maintained at $40{ }^{\circ} \mathrm{C}$. The mobile phase consisted of acetonitrile (mobile phase B) and $5 \mathrm{mM} \mathrm{NH}_{4} \mathrm{Ac}$ and $0.1 \% \mathrm{FA}$ (mobile phase A). The flow rate was maintained at $0.4 \mathrm{~mL} \mathrm{~min}{ }^{-1}$. A gradient elution program was performed as follows: $0-2.2 \mathrm{~min}$, $48 \%$ B; 2.2-2.3 min, $48-95 \%$ B; 2.3-3.3 min, $95 \%$ B; 3.3-3.4 min, $95-48 \%$ B; 3.4-4.5 min, 48\% B. The injection volume was $5.00 \mu \mathrm{L}$ and the autosampler temperature was set at $8{ }^{\circ} \mathrm{C}$.

The MS/MS detection was carried out in positive ESI mode using multiple reaction monitoring (MRM) mode. The transitions and respective mass spectrometric parameters for each analyte and internal standards are listed in Table 1. The source parameters were optimized as follows: curtain gas (CUR), $30 \mathrm{psi}$; collision activated dissociation (CAD), 7 units; nebulizer gas (GS1), 55 psi; heater gas (GS2), $60 \mathrm{psi}$; temperature, $650{ }^{\circ} \mathrm{C}$; and ion spray voltage, $4000 \mathrm{~V}$. Analyst 1.6.3 software (Applied Biosystems/Sciex, USA) was used for the instrument control, data acquisition, and processing.

\section{Preparation of stock solutions and working solutions}

The stock solution of analytes and internal standards were separately prepared in acetonitrile:dimethylsulfoxide $(1: 9, \mathrm{v} / \mathrm{v})$. The stock solutions of analytes were further serially diluted with acetonitrile:water $(1: 1, \mathrm{v} / \mathrm{v})$ to get a series of mixed working solutions containing three analytes. The working solution of the internal standard

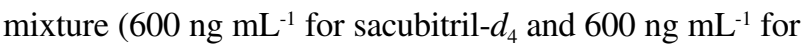
valsartan- $d_{3}$ ) was prepared in the same diluent. All of the stock solutions and working solutions were stored at $-20^{\circ} \mathrm{C}$.

Preparation of calibration standards and quality control samples

Calibration standards were prepared by spiking the working solutions with blank plasma at the ratio of 1:20 to obtain serial concentrations of $2.00,4.00,20.0,100$, $500,2000,3200$ and $4000 \mathrm{ng} \mathrm{mL}^{-1}$ of sacubitril and 5.00, $10.0,50.0,250,1250,5000,8000$ and $10000 \mathrm{ng} \mathrm{mL}^{-1}$ of valsartan and LBQ657. Quality control (QC) samples were spiked in the same way as the calibration standards at concentration levels of: $2.00 \mathrm{ng} \mathrm{mL}^{-1}$ (lower limit of quantitation QC, LLOQ QC), $6.00 \mathrm{ng} \mathrm{mL}^{-1}$ (low QC, LQC), $1600 \mathrm{ng} \mathrm{mL}^{-1}$ (medium QC, MQC), $3000 \mathrm{ng} \mathrm{mL}^{-1}$ (high QC, HQC) and $8000 \mathrm{ng} \mathrm{mL}^{-1}$ (dilution QC, DQC) of sacubitril and $5.00 \mathrm{ng} \mathrm{mL}^{-1}$ (LLOQ QC), $15.00 \mathrm{ng} \mathrm{mL}^{-1}$

Table 1. Multiple reaction monitoring (MRM) parameters of sacubitril, valsartan, LBQ657, sacubitril- $d_{4}$ and valsartan- $d_{3}$

\begin{tabular}{|c|c|c|c|c|c|c|}
\hline Analyte & Precursor ion / Da & Product ion / Da & $\mathrm{DP} / \mathrm{V}$ & $\mathrm{EP} / \mathrm{V}$ & $\mathrm{CE} / \mathrm{V}$ & $\mathrm{CXP} / \mathrm{V}$ \\
\hline Sacubitril & 412.3 & 266.2 & 51 & 11 & 28 & 18 \\
\hline Valsartan & 436.4 & 235.2 & 45 & 12 & 25 & 36 \\
\hline LBQ657 & 384.3 & 266.3 & 128 & 12 & 30 & 15 \\
\hline Sacubitril- $d_{4}$ & 416.3 & 266.2 & 57 & 11 & 21 & 20 \\
\hline Valsartan- $d_{3}$ & 439.5 & 294.2 & 65 & 5 & 24 & 35 \\
\hline
\end{tabular}

DP: declustering potential; EP: entrance potential; CE: collision energy; CXP: collision cell exit potential. 
(LQC), $4000 \mathrm{ng} \mathrm{mL}^{-1}$ (MQC), $7500 \mathrm{ng} \mathrm{mL}^{-1}$ (HQC) and $20000 \mathrm{ng} \mathrm{mL}^{-1}$ (DQC) of valsartan and LBQ657.

\section{Sample preparation}

A protein precipitation procedure was performed for plasma sample clean-up. After the addition of $25.0 \mu \mathrm{L}$ internal standards working solution into $50.0 \mu \mathrm{L}$ of plasma sample, $300 \mu \mathrm{L}$ of acetonitrile were added as a protein precipitating agent. The mixture was vortexed for $10 \mathrm{~min}$, followed by centrifugation at 2,500 $\mathrm{g}$ for $10 \mathrm{~min}$. An aliquot of $100 \mu \mathrm{L}$ supernatant was transferred to another clean 96-well plate containing $100 \mu \mathrm{L}$ acetonitrile-water $(1: 1, \mathrm{v} / \mathrm{v})$. After vortexed well for $5 \mathrm{~min}, 3.00 \mu \mathrm{L}$ of the mixture were injected into the LC-MS/MS for analysis.

\section{Application}

The developed LC-MS/MS method was applied to the simultaneous quantification of sacubitril, valsartan and LBQ657 to support a clinical pharmacokinetic study after administration of a commercial sacubitril-valsartan tablet (sacubitril: $97 \mathrm{mg}$; valsartan: $103 \mathrm{mg}$ ) in 12 healthy Chinese volunteers. This study was approved by the Research Ethics Committees of Jiangsu Province Hospital of Chinese Medicine under No. 2020L01582. All subjects gave informed consent. Blood samples were collected at the following time points: $0 \mathrm{~min}$ (pre-dose), 10 and $20 \mathrm{~min}$ and $0.5,0.75,1$, $1.5,2,2.5,3,3.5,4,5,7,9,12,24,36,48 \mathrm{~h}$ using a $4 \mathrm{~mL}$ blood-collecting tube ( $\mathrm{K}_{2}$-EDTA anticoagulant). After gently inverted for 4-5 times, the collection tubes were centrifuged at $1,700 \mathrm{~g}$ for $10 \mathrm{~min}$ at $4{ }^{\circ} \mathrm{C}$. The plasma samples separated and stored at $-70{ }^{\circ} \mathrm{C}$ until analysis.

\section{Results and Discussion}

\section{Method development}

Mass spectrometer and liquid chromatography

All analytes and internal standards showed strong response intensity and stability in positive ionization mode. Figure 1 shows the product ion spectra of $[\mathrm{M}+\mathrm{H}]^{+}$precursor ions of analytes along with their proposed fragmentation patterns. After ascertaining the precursor ions and product ions, the MS/MS parameters were optimized to maximize the response for the analytes. To produce the best sensitivity and peak shape, $0.1 \% \mathrm{FA}$ was added to promote the formation of $[\mathrm{M}+\mathrm{H}]^{+}$, and $5 \mathrm{mMNH}_{4} \mathrm{Ac}$ was added to obtain symmetrical peak shape to the sacubitril. The use of acetonitrile over methanol as the organic portion of the mobile phase has shown significant improvement in the signal response of analytes.

\section{Sample preparation}

The procedure of sample preparation in the reported literature was complicated and time consuming with liquid-liquid extraction. ${ }^{12,13}$ The study adopted simpler protein precipitation without dryness. Sacubitril- $d_{4}$ and valsartan- $d_{3}$ were selected as the internal standard of sacubitril and LBQ657, and valsartan- $d_{3}$ was selected as the internal standard of valsartan, because they had similar retention action, ionization action and extraction efficiency. Acetonitrile and methanol were taken into consideration to serve as precipitants. As shown in Figure S1 (in the Supplementary Information (SI) section), acetonitrile showed better precipitation efficiency than methanol, with lower background noise and less interference.

\section{Method validation}

\section{Specificity, linearity and LLOQ}

Six individual blank plasma samples were processed in order to investigate the potential interference from endogenous components. Cross analytes and internal standard interference were determined with samples in three replicates that were singly spiked with each analyte at upper limit of quantification (ULOQ) concentration or internal standards at nominal working concentration, respectively. There was no significant interference at retention times of the analytes and internal standards. The method was linear over the concentration range of $2.00-4000 \mathrm{ng} \mathrm{mL}^{-1}$
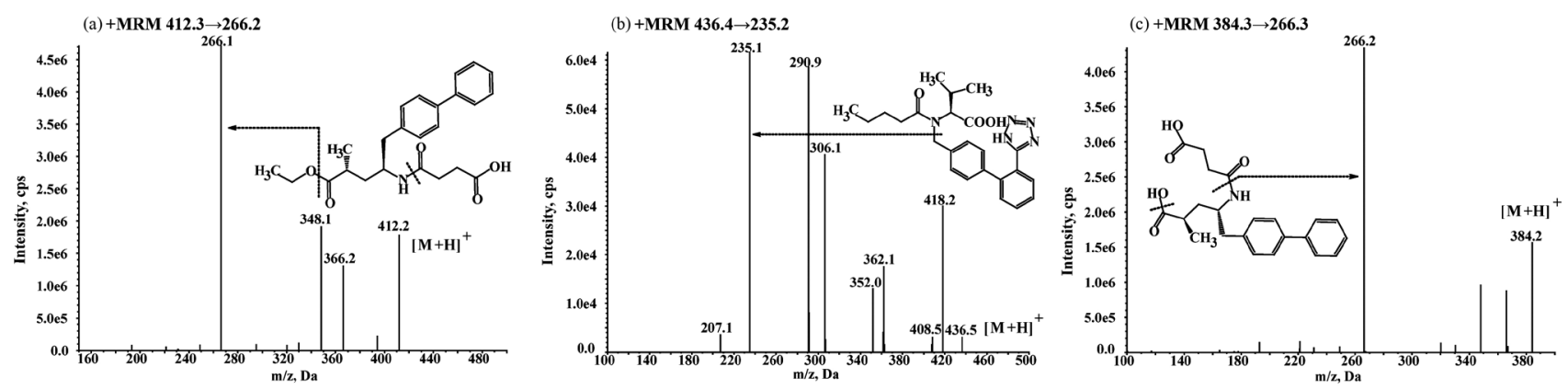

Figure 1. Positive product ion mass spectra of (a) sacubitril, (b) valsartan and (c) LBQ657. 
for sacubitril, and 5.00-10000 ng mL ${ }^{-1}$ for valsartan and LBQ657. The correlation coefficients of the calibration curves for three analytes were higher than 0.99 , indicating the excellent linearity over these concentrations. The representative standard curves of analytes are shown in Figure S2 (in the SI section). The LLOQ values of three analytes were with acceptable precision $(\leq 20.0 \%)$ and accuracy (within $\pm 20.0 \%$ ). Representative MRM chromatograms of a blank human plasma, a LLOQ sample and a $2.5 \mathrm{~h}$ plasma sample from a healthy Chinese volunteer after administration of commercial sacubitril-valsartan tablet are shown in Figure 2.

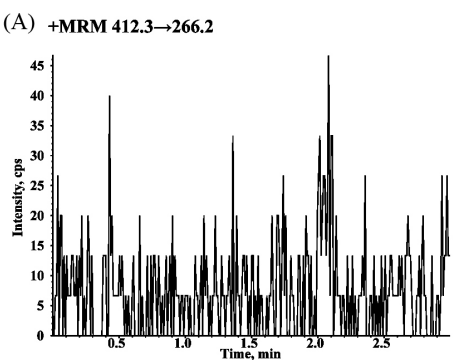

(B) + MRM $436.4 \rightarrow \mathbf{2 3 5 . 2}$

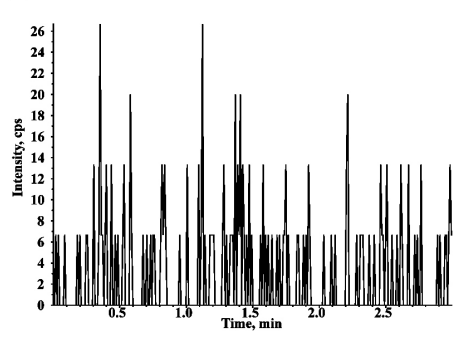

(C) + MRM 384.3 $\rightarrow 266.3$

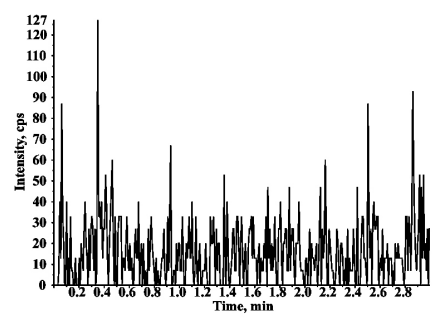

(D) + MRM 416.3 $\rightarrow 266.2$

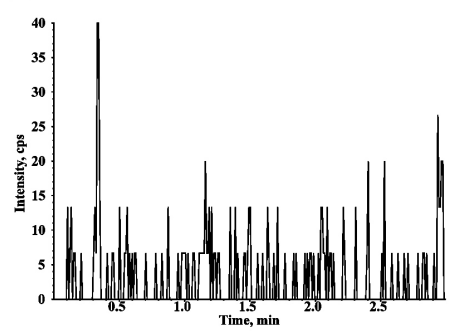

(E) + MRM 439.5 $\rightarrow 294.2$

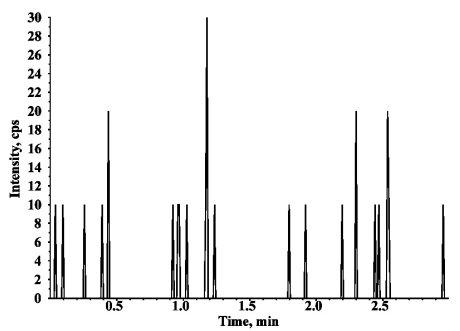

(a)

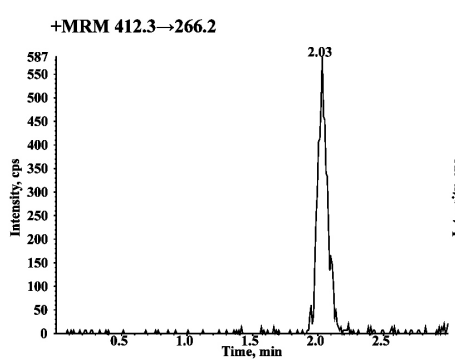

+ MRM 436.4 $\rightarrow$ 235.2
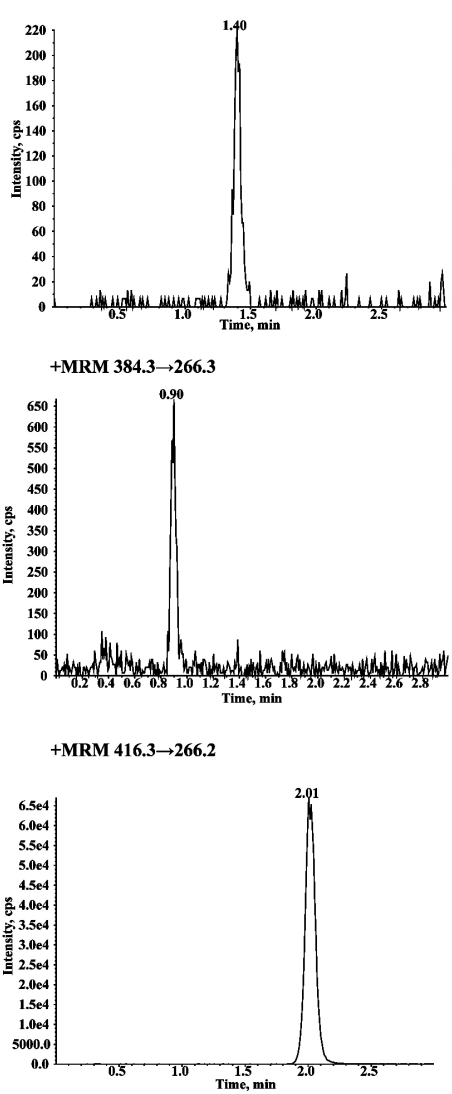

+ MRM 439.5 $\rightarrow 294.2$

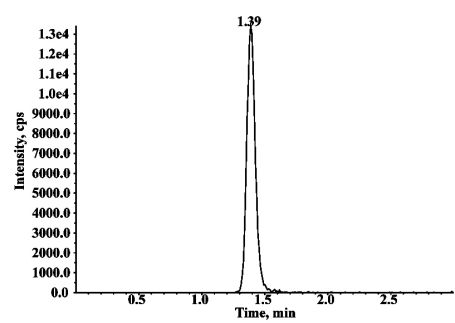

(b)

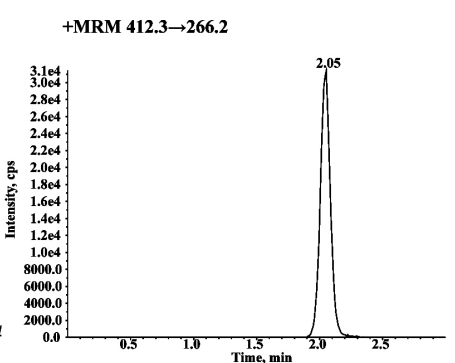

+ MRM $436.4 \rightarrow 235.2$
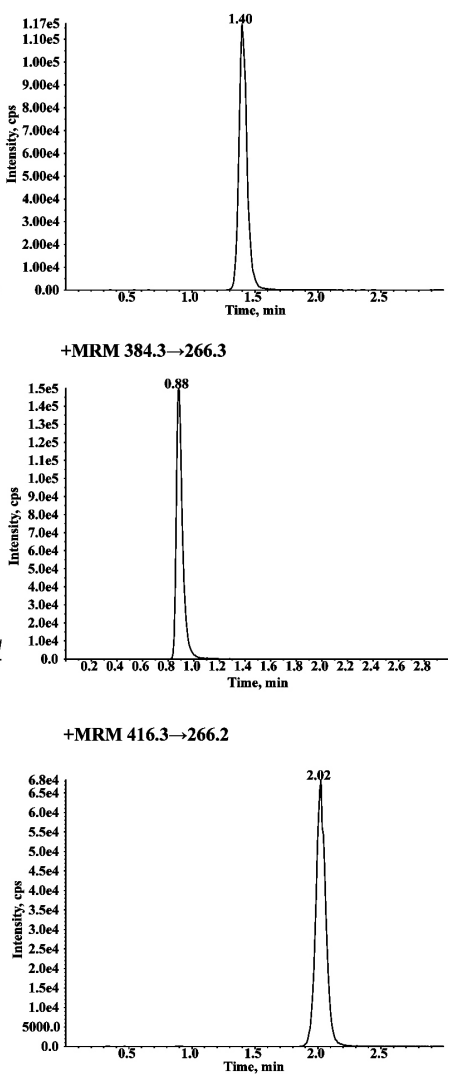

+ MRM $439.5 \rightarrow 294.2$

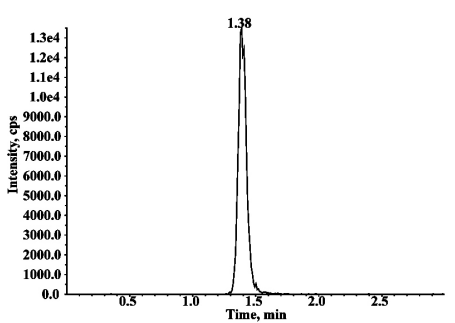

(c)

Figure 2. Representative MRM chromatograms of (A) sacubitril, (B) valsartan, (C) LBQ657, (D) sacubitril- $d_{4}$ and (E) valsartan- $d_{3}$ : (a) double blank sample, (b) LLOQ sample, and (c) $2.5 \mathrm{~h}$ plasma sample from a healthy Chinese volunteer after administration of commercial sacubitril-valsartan tablet. 


\section{Accuracy and precision}

The intra- and inter-batch accuracy and precision were assessed by analyzing six replicates of QC samples at four concentrations (LLOQ QC, LQC, MQC and HQC) in a single batch and over three batches on separate days. As summarized in Table 2, the accuracy and precision of analytes were all were within the acceptance limits.

\section{Recovery and matrix effect}

Recovery of analytes and internal standards were evaluated to ensure efficiency and reproducibility of the extraction process. The recovery of analytes and internal standards were separately determined by the response ratio of extracted samples to unextracted samples. Matrix effect was evaluated to determine the effect of the matrix on ion suppression or ion enhancement. Internal standardnormalized matrix factors were adopted to assess the matrix effect. It indicated that there was no significant impact on the determination of analytes in human plasma. The recovery and matrix data are presented in
Table 3. In addition, the calculated percent bias from determined concentrations to theoretical concentrations in hemolyzed (5\% frozen whole blood in plasma) and lipemic (20 mg mL $\mathrm{m}^{-1}$ intralipid emulsion in plasma) LQC and $\mathrm{HQC}$ samples were $\leq 5.2 \%$, indicating that there was no significant impact on the determination of analytes in the hemolyzed or lipemic plasma samples.

\section{Dilution integrity and carryover}

In order to handle the situation that a few study samples might exceed the maximum calibrated concentration, 5-fold dilution factor was evaluated by diluting QC samples at two concentrations (HQC and DQC) with blank plasma in six replicates. The accuracy and precision of DQC samples were $\leq 4.3 \%$, suggesting that the upper limit of three analytes can be extended up to the concentration levels of DQC. Double blank samples were analyzed directly following the ULOQ samples to determine carryover effects. No significant carryover peaks were observed at the retention times of the analytes and internal standards.

Table 2. Intra- and inter-batch precision and accuracy data of sacubitril, valsartan and LBQ657 in human plasma

\begin{tabular}{|c|c|c|c|c|c|c|c|}
\hline \multirow[b]{2}{*}{ Analyte } & \multirow{2}{*}{$\begin{array}{c}\text { Nominal } \\
\text { concentration / } \\
\left(\mathrm{ng} \mathrm{mL} \mathrm{mL}^{-1}\right)\end{array}$} & \multicolumn{3}{|c|}{ Intra-batch $(\mathrm{n}=6)$} & \multicolumn{3}{|c|}{ Inter-batch $(n=6 \times 3)$} \\
\hline & & $\begin{array}{l}\text { Measured concentration } \\
\text { mean } \pm \mathrm{SD} /\left(\mathrm{ng} \mathrm{mL}^{-1}\right)\end{array}$ & $\begin{array}{l}\text { Accuracy } \\
\text { RE / \% }\end{array}$ & $\begin{array}{l}\text { Precision } \\
\mathrm{CV} / \%\end{array}$ & $\begin{array}{l}\text { Measured concentration } \\
\text { mean } \pm \mathrm{SD} /\left(\mathrm{ng} \mathrm{mL}^{-1}\right)\end{array}$ & $\begin{array}{l}\text { Accuracy } \\
\mathrm{RE} / \%\end{array}$ & $\begin{array}{c}\text { Precision } \\
\mathrm{CV} / \%\end{array}$ \\
\hline \multirow{4}{*}{ Sacubitril } & 2.00 (LLOQ QC) & $1.75 \pm 0.05$ & -12.7 & 3.1 & $1.79 \pm 0.16$ & -10.3 & 9.2 \\
\hline & 6.00 (LQC) & $5.66 \pm 0.51$ & -5.7 & 9.0 & $5.87 \pm 0.54$ & -2.2 & 9.2 \\
\hline & 1600 (MQC) & $1478 \pm 108$ & -7.6 & 7.3 & $1484 \pm 94$ & -7.3 & 6.3 \\
\hline & 3000 (HQC) & $2842 \pm 141$ & -5.3 & 5.0 & $2768 \pm 143$ & -7.7 & 5.2 \\
\hline \multirow{4}{*}{ Valsartan } & 5.00 (LLOQ QC) & $5.22 \pm 0.27$ & 4.3 & 5.2 & $4.95 \pm 0.44$ & -1.1 & 8.9 \\
\hline & $15.0(\mathrm{LQC})$ & $15.6 \pm 0.9$ & 4.2 & 5.9 & $15.2 \pm 1.2$ & 1.3 & 7.7 \\
\hline & 4000 (MQC) & $3947 \pm 153$ & -1.3 & 3.9 & $3951 \pm 116$ & -1.2 & 2.9 \\
\hline & 7500 (HQC) & $7143 \pm 255$ & -4.8 & 3.6 & $7108 \pm 273$ & -5.2 & 3.8 \\
\hline \multirow{4}{*}{ LBQ657 } & 5.00 (LLOQ QC) & $5.14 \pm 0.35$ & 2.9 & 6.9 & $5.74 \pm 0.74$ & 14.8 & 12.9 \\
\hline & 15.0 (LQC) & $14.1 \pm 0.5$ & -5.8 & 3.8 & $15.2 \pm 1.0$ & 1.1 & 6.9 \\
\hline & 4000 (MQC) & $3874 \pm 47$ & -3.1 & 1.2 & $3952 \pm 107$ & -1.2 & 2.7 \\
\hline & 7500 (HQC) & $7345 \pm 101$ & -2.1 & 1.4 & $7537 \pm 257$ & 0.5 & 3.4 \\
\hline
\end{tabular}

SD: standard deviation; RE: relative error; CV: coefficient of variation; n: number of replicates; QC: quality control; LLOQ: lower limit of quantitation; LQC: low QC; MQC: medium QC; HQC: high QC.

Table 3. Recovery and matrix effect of sacubitril, valsartan and LBQ657 in human plasma

\begin{tabular}{|c|c|c|c|c|c|}
\hline \multirow{2}{*}{ Analyte } & \multirow{2}{*}{$\begin{array}{c}\text { Nominal concentration / } \\
(\mathrm{ng} \mathrm{mL}-1)\end{array}$} & \multicolumn{2}{|c|}{ Recovery $(n=6)$} & \multicolumn{2}{|c|}{ Matrix effect $(n=6)$} \\
\hline & & Mean value / \% & $\mathrm{CV} / \%$ & Mean value / \% & $\mathrm{CV} / \%$ \\
\hline \multirow{3}{*}{ Sacubitril } & 6.00 (LQC) & 93.3 & 3.7 & 99.9 & 3.5 \\
\hline & 1600 (MQC) & 97.4 & 4.5 & 100.9 & 2.8 \\
\hline & $3000(\mathrm{HQC})$ & 98.1 & 4.6 & 102.5 & 3.5 \\
\hline \multirow{3}{*}{ Valsartan } & $15.0(\mathrm{LQC})$ & 77.8 & 9.5 & 99.3 & 3.6 \\
\hline & 4000 (MQC) & 79.3 & 4.0 & 106.0 & 1.5 \\
\hline & 7500 (HQC) & 78.4 & 4.6 & 98.6 & 1.5 \\
\hline \multirow{3}{*}{ LBQ657 } & 15.0 (LQC) & 54.6 & 1.6 & 94.8 & 2.3 \\
\hline & 4000 (MQC) & 54.2 & 3.1 & 94.5 & 2.6 \\
\hline & $7500(\mathrm{HQC})$ & 53.4 & 7.8 & 91.1 & 1.7 \\
\hline
\end{tabular}

CV: coefficient of variation; n: number of replicates; QC: quality control; LQC: low QC; MQC: medium QC; HQC: high QC. 


\section{Stability}

The stability of analytes was evaluated under a variety of storage and process conditions. Stock solution of sacubitril, valsartan, and LBQ657 are stable for $16.6 \mathrm{~h}$ at room temperature and for 67 days at $-20^{\circ} \mathrm{C}$. The stability of analytes in plasma and supernatant was summarized in Table 4. The stability of analytes in human blood with $\mathrm{K}_{2}$-EDTA as anticoagulant was performed with the centrifugal parameters of $1,700 \mathrm{~g}, 10 \mathrm{~min}$, and $4{ }^{\circ} \mathrm{C}$. Three analytes were stable in blood for 1 and $2 \mathrm{~h}$ at room temperature.

\section{Pharmacokinetic study}

The validated LC-MS/MS method was used to determine the concentrations of sacubitril, valsartan, and LBQ657 after single oral administration of commercial sacubitril-valsartan tablet. The mean plasma concentrationtime profile of sacubitril, valsartan, and LBQ657 in Chinese healthy volunteers $(n=12)$ are presented in Figure 3, and the calculated pharmacokinetic parameters are shown in Table 5. Those pharmacokinetics parameters in Caucasian and Japanese subjects, which were reported

Table 4. Stability of sacubitril, valsartan and LBQ657 in human plasma under various storage conditions $(n=3)$

\begin{tabular}{|c|c|c|c|c|c|c|c|c|c|}
\hline \multirow[b]{2}{*}{ Storage condition } & \multicolumn{3}{|c|}{ Sacubitril } & \multicolumn{3}{|c|}{ Valsartan } & \multicolumn{3}{|c|}{ LBQ657 } \\
\hline & $\begin{array}{c}\text { Nominal } \\
\text { concentration / } \\
\left(\mathrm{ng} \mathrm{mL} \mathrm{mL}^{-1}\right)\end{array}$ & $\mathrm{RE} / \%$ & $\mathrm{CV} / \%$ & $\begin{array}{c}\text { Nominal } \\
\text { concentration / } \\
\left(\mathrm{ng} \mathrm{mL} \mathrm{mL}^{-1}\right)\end{array}$ & $\mathrm{RE} / \%$ & $\mathrm{CV} / \%$ & $\begin{array}{c}\text { Nominal } \\
\text { concentration / } \\
\left(\mathrm{ng} \mathrm{mL} \mathrm{mL}^{-1}\right)\end{array}$ & $\mathrm{RE} / \%$ & $\mathrm{CV} / \%$ \\
\hline Room temperature stability & $6.00(\mathrm{LQC})$ & -7.7 & 4.6 & $15.0(\mathrm{LQC})$ & 8.1 & 3.4 & $15.0(\mathrm{LQC})$ & -1.4 & 2.0 \\
\hline$\left(25.0^{\circ} \mathrm{C}, 22.8 \mathrm{~h}\right)$ & 3000 (HQC) & -1.0 & 3.0 & $7500(\mathrm{HQC})$ & -2.5 & 1.6 & $7500(\mathrm{HQC})$ & -0.5 & 0.2 \\
\hline Freeze-thaw cycles & $6.00(\mathrm{LQC})$ & 1.4 & 3.4 & $15.0(\mathrm{LQC})$ & -8.4 & 5.7 & $15.0(\mathrm{LQC})$ & 8.4 & 4.1 \\
\hline$\left(-20^{\circ} \mathrm{C}\right.$, five cycles $)$ & $3000(\mathrm{HQC})$ & 0.1 & 3.8 & $7500(\mathrm{HQC})$ & 1.1 & 9.4 & $7500(\mathrm{HQC})$ & 3.2 & 2 \\
\hline Autosampler stability & $6.00(\mathrm{LQC})$ & 1.8 & 1.6 & $15.0(\mathrm{LQC})$ & -7.0 & 0.9 & $15.0(\mathrm{LQC})$ & 7.6 & 1.5 \\
\hline ( $8^{\circ} \mathrm{C}, 4$ days $)$ & 3000 (HQC) & 6.3 & 5.3 & 7500 (HQC) & -0.4 & 0.8 & $7500(\mathrm{HQC})$ & 4.5 & 3.4 \\
\hline Long-term stability & $6.00(\mathrm{LQC})$ & -8.8 & 2.4 & $15.0(\mathrm{LQC})$ & 5.8 & 3.0 & $15.0(\mathrm{LQC})$ & -0.6 & 8.4 \\
\hline$\left(-20^{\circ} \mathrm{C}, 50\right.$ days $)$ & $3000(\mathrm{HQC})$ & 5.6 & 1.2 & $7500(\mathrm{HQC})$ & 5.5 & 3.6 & $7500(\mathrm{HQC})$ & 4.2 & 2.1 \\
\hline
\end{tabular}

RE: relative error; CV: coefficient of variation; n: number of replicates; QC: quality control; LQC: low QC; HQC: high QC.
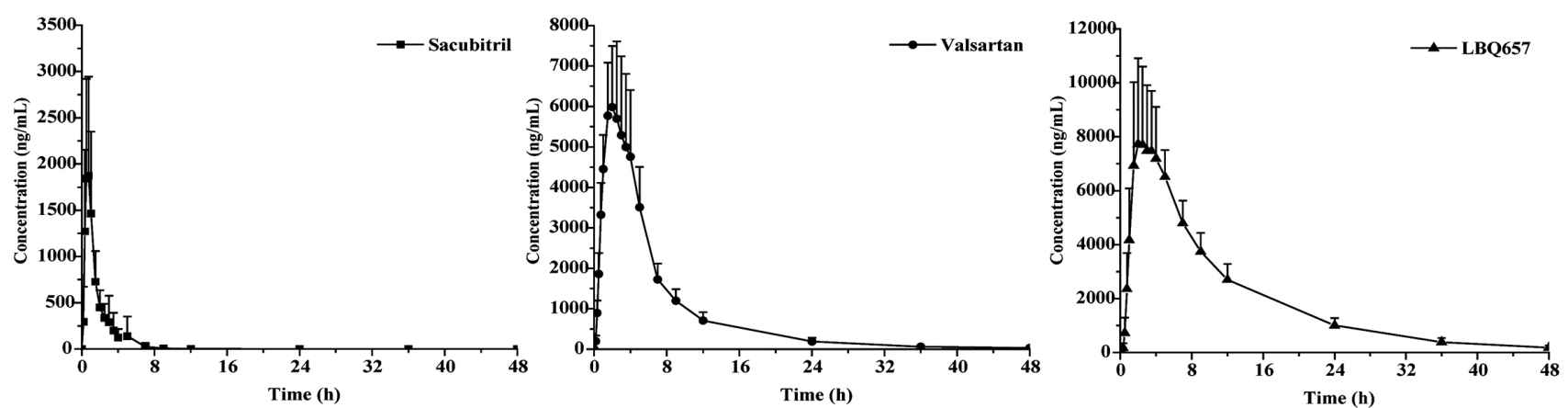

Figure 3. Mean plasma concentration-time profiles of sacubitril, valsartan and LBQ657 after administration of commercial sacubitril-valsartan tablet (data are means $\pm \mathrm{SD}, \mathrm{n}=12$ ).

Table 5. The summary and comparison of pharmacokinetic parameters of sacubitril, valsartan and LBQ657 measured in healthy volunteers after oral administration of sacubitril-valsartan tablet $(200 \mathrm{mg})$

\begin{tabular}{|c|c|c|c|c|c|c|c|c|c|}
\hline \multirow{2}{*}{ Parameter } & \multicolumn{3}{|c|}{ Chinese subjects in this study $(\mathrm{n}=12)$} & \multicolumn{3}{|c|}{ Caucasian subjects $^{\mathrm{a}}(\mathrm{n}=8)$} & \multicolumn{3}{|c|}{ Janpanese subjects $^{\mathrm{b}}(\mathrm{n}=8)$} \\
\hline & Sacubitril & Valsartan & LBQ657 & Sacubitril & Valsartan & LBQ657 & Sacubitril & Valsartan & LBQ657 \\
\hline $\mathrm{C}_{\max } /\left(\mathrm{ng} \mathrm{mL}^{-1}\right)$ & $2580 \pm 1058$ & $5929 \pm 1662$ & $8873 \pm 1929$ & $1974 \pm 678$ & $3990 \pm 685$ & $8529 \pm 1659$ & $2470 \pm 923$ & $3981 \pm 1392$ & $8482 \pm 1541$ \\
\hline $\mathrm{T}_{\max } / \mathrm{h}$ & $0.9 \pm 0.8$ & $2.1 \pm 0.9$ & $3.2 \pm 1.3$ & $0.6 \pm 0.2$ & $1.8 \pm 0.5$ & $1.8 \pm 0.5$ & $0.50 \pm 0.5$ & $1.8 \pm 0.8$ & $2.1 \pm 0.6$ \\
\hline $\mathrm{t}_{1 / 2} / \mathrm{h}$ & $1.3 \pm 0.3$ & $8.6 \pm 2.3$ & $9.1 \pm 1.3$ & $1.7 \pm 0.9$ & $22.1 \pm 16.5$ & $13.01 \pm 3.0$ & $1.9 \pm 1.0$ & $18.9 \pm 7.4$ & $13.4 \pm 0.9$ \\
\hline $\mathrm{AUC}_{\text {last }} /\left(\mathrm{h} \mathrm{ng} \mathrm{mL} \mathrm{mL}^{-1}\right)$ & $3038 \pm 678$ & $37833 \pm 10119$ & $93267 \pm 13389$ & $2083 \pm 803$ & $24097 \pm 4680$ & $82903 \pm 16136$ & $2580 \pm 325$ & $22076 \pm 6691$ & $71635 \pm 10533$ \\
\hline $\mathrm{AUC}_{0-\infty} /\left(\mathrm{h} \mathrm{ng} \mathrm{mL} L^{-1}\right)$ & $3042 \pm 678$ & $38233 \pm 10313$ & $95714 \pm 14402$ & $2087 \pm 803$ & $21079 \pm 4087$ & $70450 \pm 12623$ & $2580 \pm 325$ & $22180 \pm 6673$ & $71775 \pm 13098$ \\
\hline $\mathrm{CL} /\left(\mathrm{mL} \mathrm{h}^{-1}\right)$ & $33676 \pm 8836$ & $2914 \pm 886$ & $\mathrm{NC}$ & - & - & - & - & - & - \\
\hline $\mathrm{V}_{\mathrm{d}} / \mathrm{mL}$ & $61987 \pm 17799$ & $35773 \pm 2914$ & $\mathrm{NC}$ & - & - & - & - & - & - \\
\hline
\end{tabular}

Pharmacokinetic parameters from the studies of ${ }^{\mathrm{a}} \mathrm{Gu}$ et al..$^{12}$ in Caucasian subjects and ${ }^{\mathrm{b}} \mathrm{Akahori}$ et al. ${ }^{13}$ in Japanese subjects; n: number of subjects; $\mathrm{C}_{\mathrm{max}}$ : the maximum plasma concentration; $\mathrm{T}_{\max }$ : the time to reach the $\mathrm{C}_{\max } ; \mathrm{t}_{1 / 2}$ : elimination half-life time; $\mathrm{AUC}_{\text {last }}$ : area under the plasma concentration-time curve from zero to last hour; $\mathrm{AUC}_{0-\infty}: \mathrm{AUC}_{0-\mathrm{t}}$ extrapolated to infinity; $\mathrm{V}_{\mathrm{d}}$ : apparent volume of distribution; $\mathrm{CL}$ : the plasma clearance; NC: not calculated, since LBQ657 is the metabolite of sacubitril and no dose information is available. 
in previous studies, ${ }^{12,13}$ were also summarized in Table 5. The $\mathrm{T}_{\max }$ (the time to reach the $\mathrm{C}_{\max }$, the maximum plasma concentration) and $t_{1 / 2}$ (elimination half-life time) values of sacubitril, valsartan and LBQ657 obtained in this study were similar to those in the previous studies in Caucasian and Japanese subjects. However, the AUC (area under the plasma concentration-time curve) and $\mathrm{C}_{\max }$ values in Chinese subjects were higher than those Caucasian and Japanese subjects, which may offer valuable information in promoting rational drug use of sacubitril-valsartan tablets in Chinese subjects.

\section{Conclusions}

For the first time, a rapid and sensitive LC-MS/MS method was developed and fully validated for the stimulation simultaneous determination of sacubitril, valsartan and LBQ657 in human plasma. The concentrations of sacubitril, valsartan and LBQ657 in human plasma were analyzed reliably by simple protein precipitation procedure. Comprehensive method validations were all conducted following FDA guidance. Furthermore, the reproducible LC-MS/MS method was successfully applied to a pharmacokinetic study in Chinese subjects. The difference of AUC and $\mathrm{C}_{\max }$ values in Chinese subjects, Caucasian and Japanese subjects may also provide reference for clinical rational use of the drug.

\section{Supplementary Information}

Supplementary file is available free of charge at https://jbcs.sbq.org.br as PDF file.

\section{Acknowledgments}

The authors thank Shunbo Zhao, Keli Wang and Xiaojie Ye from Nanjing Clinical Technical Laboratories Inc., China for their help during the research.

\section{Author Contributions}

Yufeng $\mathrm{Ni}$ was responsible for conceptualization, methodology, validation, formal analysis, investigation, writing-original draft and writing-review and editing; Yujia Zhang for methodology, validation, formal analysis, investigation, writing-original draft and writing-review and editing; Chong Zou for conceptualization, resources, supervision, project administration, writing-review and editing; Li Ding for conceptualization, methodology, validation, formal analysis, investigation, resources, writingreview and editing, supervision and project administration.

\section{References}

1. Nielsen, E. E.; Feinberg, J. B.; Bu, F.-L.; Hecht Olsen, M.; Raymond, I.; Steensgaard-Hansen, F.; Jakobsen, J. C.; Open Heart 2020, 7, e001294.

2. Roger, V. L.; Circ. Res. 2013, 113, 646.

3. Liu, L.; Eisen, H. J.; Cardiol. Clin. 2014, 32, 1.

4. Cook, C.; Cole, G.; Asaria, P.; Jabbour, R.; Francis, D. P.; Int. J. Cardiol. 2014, 171, 368.

5. McMurray, J. J. V.; Packer, M.; Desai, A. S.; Gong, J.; Lefkowitz, M. P.; Rizkala, A. R.; Rouleau, J.; Shi, V. C.; Solomon, S. D.; Swedberg, K.; Zile, M. R.; Eur. J. Heart Fail 2013, 15, 1062.

6. Kaplinsky, E.; Ther. Adv. Chronic Dis. 2016, 7, 278.

7. Ayalasomayajula, S.; Langenickel, T.; Pal, P.; Boggarapu, S.; Sunkara, G.; Clin. Pharmacokinet. 2017, 56, 1461.

8. Hanna, I.; Alexander, N.; Crouthamel, M. H.; Davis, J.; Natrillo, A.; Tran, P.; Vapurcuyan, A.; Zhu, B.; Xenobiotica 2018, 48, 300.

9. Kobalava, Z.; Kotovskaya, Y.; Averkov, O.; Pavlikova, E.; Moiseev, V.; Albrecht, D.; Chandra, P.; Ayalasomayajula, S.; Prescott, M. F.; Pal, P.; Langenickel, T. H.; Jordaan, P.; Rajman, I.; Cardiovasc. Ther. 2016, 34, 191.

10. Attimarad, M.; Nagaraja, S. H.; Nair, A. B.; Aldhubaib, B. E.; Katharigatta, V. N.; J. Liq. Chromatogr. Related Technol. 2018, $41,246$.

11. Chunduri, R. H. B.; Dannana, G. S.; Biomed. Chromatogr. 2016, 30, 1467.

12. Gu, J.; Noe, A.; Chandra, P.; Al-Fayoumi, S.; Ligueros-Saylan, M.; Sarangapani, R.; Maahs, S.; Ksander, G.; Rigel, D. F.; Jeng, A. Y.; Lin, T. H.; Zheng, W.; Dole, W. P.; J. Clin. Pharmacol. 2010, 50, 401.

13. Akahori, M.; Ayalasomayajula, S.; Langenickel, T.; Pal, P.; Zhou, W.; Sunkara, G.; Eur. J. Drug Metab. Pharmacokinet. 2017, 42, 407.

14. Han, Y.; Ayalasomayajula, S.; Pan, W.; Yang, F.; Yuan, Y.; Langenickel, T.; Hinder, M.; Kalluri, S.; Pal, P.; Sunkara, G.; Eur. J. Drug Metab. Pharmacokinet. 2017, 42, 109.

15. Food and Drug Administration (FDA); Guidance for Industry: Bioanalytical Method Validation, available at https://www.fda. gov/regulatory-information/search-fda-guidance-documents/ bioanalytical-method-validation-guidance-industry, accessed on March 25, 2021.

Submitted: February 11, 2021

Published online: May 7, 2021 